\title{
Estimates for the Stokes Operator in Lipschitz Domains
}

\author{
Russell M. Brown \& Zhongwei Shen
}

\begin{abstract}
We study the Stokes operator $A$ in a threedimensional Lipschitz domain $\Omega$. Our main result asserts that the domain of $A$ is contained in $W_{0}^{1, p}(\Omega) \cap W^{3 / 2,2}(\Omega)$ for some $p>3$. Certain $L^{\infty}$-estimates are also established. Our results may be used to improve the regularity of strong solutions of Navier-Stokes equations in nonsmooth domains. In the appendix we provide a simple proof of area integral estimates for solutions of Stokes equations.
\end{abstract}

Introduction. In a recent interesting paper, Deuring and von Wahl [DW] consider strong solutions of the nonstationary Navier-Stokes equations in $\Omega \times$ $(0, T)$ :

$$
\left\{\begin{array}{l}
\frac{\partial u}{\partial t}=\Delta u-(u \cdot \nabla) u-\nabla \pi+f, \\
\operatorname{div} u=0
\end{array}\right.
$$

with the initial-Dirichlet condition

$$
\left\{\begin{array}{l}
u(X, t)=0 \text { for }(X, t) \in \partial \Omega \times(0, T), \\
u(X, 0)=u_{0}(X), \quad X \in \Omega
\end{array}\right.
$$

where $\Omega$ is a bounded Lipschitz domain in $\mathbb{R}^{3}$. Based on the functional analytical approach of Fujita and Kato [FK] and the Rellich estimates of Shen [S1], they show that, if $u_{0} \in \mathcal{D}\left(A^{1 / 4+\varepsilon}\right)$ for some $\varepsilon \in\left(0, \frac{1}{2}\right)$ and $f$ is bounded and locally Hölder continuous, then a solution $(u, \pi)$ exists for some $T>0$ and

$$
u \in C((0, T], \mathcal{D}(A)) \cap C\left([0, T], \mathcal{D}\left(A^{1 / 4+\varepsilon}\right)\right),
$$

where $A=-P \Delta$ denotes the Stokes operator.

The purpose of this note is to describe $\mathcal{D}(A)$, the domain of $A$, in terms of Sobolev's spaces. In the case of smooth domains, it is well known that

$$
\mathcal{D}(A)=W^{2,2}(\Omega) \cap W_{0}^{1,2}(\Omega) \cap L_{\sigma}^{2}(\Omega)
$$


where $L_{\sigma}^{2}(\Omega)$ denotes the space of solenoidal functions in $L^{2}(\Omega)$ (e.g., see $[\mathrm{CF}]$ ). One can not expect such results in Lipschitz domains, as the $W^{2,2}$-estimate, in general, fails in nonsmooth domains. Our main results in this paper assert that

$$
\mathcal{D}(A) \subset W_{0}^{1, p}(\Omega) \cap W^{3 / 2,2}(\Omega)
$$

for some $p=p(\Omega)>3$ (Theorem 2.17). In particular, it follows from Sobolev's imbedding that for every $t \in(0, T], u(t) \in C^{\alpha}(\bar{\Omega})$ for some $\alpha=\alpha(\Omega)>0$, i.e., the strong solution of the Navier-Stokes equations is Hölder continuous up to the boundary as a function of $X$. We also obtain the following $L^{\infty}$ estimates:

$$
\begin{aligned}
& \|u\|_{L^{\infty}(\Omega)} \leq C\|\nabla u\|_{L^{2}(\Omega)}^{1 / 2}\|A u\|_{L^{2}(\Omega)}^{1 / 2}, \\
& \|u\|_{L^{\infty}(\Omega)} \leq C\|u\|_{L^{2}(\Omega)}^{1 / 4}\|A u\|_{L^{2}(\Omega)}^{3 / 4}
\end{aligned}
$$

for $u \in \mathcal{D}(A)$. To establish (0.2), we use the reverse Hölder inequality, (0.1) and some localization techniques. See Theorem 3.1 and Corollary 3.2. Estimates like $(0.2)$ are very useful in the study of Navier-Stokes equations. See $[\mathrm{CF}]$ and $[\mathrm{H}]$ in the case of smooth domains.

To prove (0.1), we shall study the Dirichlet problem for the Stokes equations with a forcing term, and interpolate between the $L^{2}$ estimates in [FKV] and the Hölder estimates in [S2]. The following area integral estimate,

$$
\int_{\Omega}|\nabla u(X)|^{2} \operatorname{dist}(X, \partial \Omega) d X \leq C \int_{\partial \Omega}|u|^{2} d \sigma
$$

for solutions of Stokes equations $\Delta u=\nabla \pi, \operatorname{div} u=0$ in $\Omega$, plays an important role. This estimate is due to E. Fabes, C. Kenig and G. Verchota, but no proof has appeared in the literature. In the appendix of this paper, which is due to Z. Shen, we will provide a simple proof of the area integral estimates for solutions of Stokes equations. The proof given here is based on the idea of a recent paper by B. Dahlberg, C. Kenig, J. Pipher, and G. Verchota [DKPV] for higher order equations and systems, together with some observations on the pressure term $\pi$.

Finally, the second author would like to thank C. Kenig for many helpful conversations.

1. Notation and definitions. In this section we collect the definitions for Lipschitz domains, the nontangential maximal function, the Sobolev and Besov spaces we will use, and the Stokes operator. We shall also recall a few elementary facts regarding complex interpolation. 
Lipschitz domains. Let $\Omega$ be a bounded, open, connected set in $\mathbb{R}^{n}$. We say that $\Omega$ is a Lipschitz domain if for each $P \in \partial \Omega$, there exists a coordinate system $\left(x^{\prime}, x_{n}\right)$, which is isometric to the usual coordinates, and a Lipschitz function $\psi: \mathbb{R}^{n-1} \rightarrow \mathbb{R}$, a radius $r>0$ so that

$$
\begin{aligned}
B(P, 2 r) \cap \Omega & =\left\{\left(x^{\prime}, x_{n}\right) \in \mathbb{R}^{n}: x_{n}>\psi\left(x^{\prime}\right)\right\} \cap B(P, 2 r), \\
B(P, 2 r) \cap \partial \Omega & =\left\{\left(x^{\prime}, x_{n}\right) \in \mathbb{R}^{n}: x_{n}=\psi\left(x^{\prime}\right)\right\} \cap B(P, 2 r) .
\end{aligned}
$$

The nontangential maximal function. For a function $u$ on $\Omega$, we define its nontangential maximal function $(u)^{*}$ by

$$
(u)^{*}(P)=\sup \{|u(X)|: X \in \Omega,|X-P| \leq 2 \operatorname{dist}(X, \partial \Omega)\}, \quad P \in \partial \Omega .
$$

We now give the definition of the function spaces we will use.

Sobolev and Besov spaces. For $\Omega \subset \mathbb{R}^{n}, p \in[1, \infty)$ and $k=1,2, \ldots$, we let $W^{k, p}(\Omega)$ denote the space of functions $u$ on $\Omega$ such that the norm

$$
\|u\|_{W^{k, p}(\Omega)} \equiv \sum_{|\alpha| \leq k}\left\|D^{\alpha} u\right\|_{L^{p}(\Omega)}
$$

is finite.

For $1 \leq p<\infty$ and $0<\alpha<1$, we define $W^{\alpha, p}(\Omega)$ to be the collection of functions $u$ on $\Omega$ with the norm

$$
\|u\|_{W^{\alpha, p}(\Omega)} \equiv\|u\|_{L^{p}(\Omega)}+\left(\iint_{\Omega \times \Omega} \frac{|u(X)-u(Y)|^{p}}{|X-Y|^{n+\alpha p}} d X d Y\right)^{1 / p}<\infty .
$$

For $1<\alpha<2$, we define $W^{\alpha, p}(\Omega)$ to be the set of functions on $\Omega$ such that

$$
\|u\|_{W^{\alpha, p}(\Omega)} \equiv\|u\|_{L^{p}(\Omega)}+\|\nabla u\|_{W^{\alpha-1, p}(\Omega)}<\infty .
$$

We may define $W^{\alpha, p}(\partial \Omega)$ for $0<\alpha<1$ and $1 \leq p<\infty$ in a similar manner with the integral over $\partial \Omega \times \partial \Omega$.

For a region $D$ above the graph of a Lipschitz function $\varphi$, i.e., $D=\left\{\left(x^{\prime}, x_{n}\right) \in\right.$ $\left.\mathbb{R}^{n}: x_{n}>\varphi\left(x^{\prime}\right)\right\}$, we define the space $W^{1,2}(\partial D)$ as the space of functions $f\left(x^{\prime}, \varphi\left(x^{\prime}\right)\right)=g\left(x^{\prime}\right)$ where $g \in W^{1,2}\left(\mathbb{R}^{n-1}\right)$. Using a partition of unity for $\partial \Omega$, we may extend this definition to the boundary of a bounded Lipschitz domain $\Omega$ for $W^{1,2}(\partial \Omega)$. We remark that $W^{\alpha, p}(\partial \Omega)$ may also be defined in this manner. See [Gr, p. 20].

We will use $W_{0}^{k, p}(\Omega)$ to denote the closure of $C_{0}^{\infty}(\Omega)$ in the norm of $W^{k, p}(\Omega)$. If $k$ is a nonnegative integer, we use $W^{k, p}(\Omega)$ to denote the dual of $W_{0}^{-k, p}(\Omega)$.

We will use the same notation $L^{p}(\Omega), W^{\alpha, p}(\Omega), W^{k, p}(\Omega)$, etc., for vectorial counterparts. 
Complex interpolation. We will need the following results on complex interpolation:

$$
\left[L^{2}(\partial \Omega), W^{1,2}(\partial \Omega)\right]_{\vartheta}=W^{\vartheta, 2}(\partial \Omega)
$$

and

$$
\left[W^{\alpha, 2}(\partial \Omega), C^{\gamma}(\partial \Omega)\right]_{\vartheta}=W^{t, p}(\partial \Omega)
$$

where

$$
\frac{1}{p}=\frac{\vartheta}{2}, \quad \text { and } \quad t=\alpha \vartheta+\gamma(1-\vartheta) .
$$

When $\partial \Omega$ is replaced by $\mathbb{R}^{n},(1.3)$ and (1.4) are well known (e.g., see [BL]). To extend this result to boundaries of Lipschitz domains, we use the following easy proposition, whose proof is omitted.

Proposition 1.5. Suppose $A_{i}, B_{i}(i=0,1)$ are Banach spaces and $\{C(\vartheta)$ : $\vartheta \in[0,1]\}$ is a family of Banach spaces such that $C(0)=A_{0}, C(1)=A_{1}$ and $C(\vartheta) \subset A_{0}+A_{1}$. Also assume that there exist linear operators $I: A_{0}+A_{1} \rightarrow$ $B_{0}+B_{1}$ and $P: B_{0}+B_{1} \rightarrow A_{0}+A_{1}$ such that $I: C(\vartheta) \rightarrow\left[B_{0}, B_{1}\right]_{\vartheta}, P:$ $\left[B_{0}, B_{1}\right]_{\vartheta} \rightarrow C(\vartheta)$ are bounded for each $\vartheta \in[0,1]$ and $P \circ I$ is the identity on $A_{0}+A_{1}$. Then

$$
\left[A_{0}, A_{1}\right]_{\vartheta}=C(\vartheta) .
$$

To see (1.3), let $\left\{B_{j}=B\left(P_{j}, r\right): j=1,2, \ldots, N\right\}$ be a covering of $\partial \Omega$ by balls as in the definition of Lipschitz domain, and $\eta_{j} \in C_{0}^{\infty}\left(\mathbb{R}^{n}\right)$ such that $\operatorname{supp} \eta_{j} \subset B_{j}, 0 \leq \eta_{j} \leq 1, \sum_{j} \eta_{j}=1$ on $\partial \Omega$. Let $\varphi_{j}$ be an isometry of $\mathbb{R}^{n}$ such that

$$
\varphi_{j}\left(B_{j} \cap \Omega\right)=\left\{\left(x^{\prime}, x_{n}\right): x_{n}>\psi_{j}\left(x^{\prime}\right),\left|\left(x^{\prime}, x_{n}\right)\right| \leq r\right\} .
$$

We now choose $A_{0}=L^{2}(\partial \Omega), A_{1}=W^{1,2}(\partial \Omega), B_{0}=L^{2}\left(\mathbb{R}^{(n-1)}, \mathbb{R}^{N}\right), B_{1}=$ $W^{1,2}\left(\mathbb{R}^{(n-1)}, \mathbb{R}^{N}\right)$, and $C(\vartheta)=W^{\vartheta, 2}(\partial \Omega)$. If $g$ is a function of $\partial \Omega$, we define $I g$ by letting the $j^{\text {th }}$ component be given by

$$
I g\left(x^{\prime}\right)_{j}=\left(\eta_{j} g\right)\left(\varphi_{j}^{-1}\left(x^{\prime}, \psi_{j}\left(x^{\prime}\right)\right)\right) .
$$

We let $\tilde{\eta}_{j} \in C_{0}^{\infty}\left(B\left(P_{j}, 2 r\right)\right), j=1, \ldots, N$, be functions satisfying $\tilde{\eta}_{j}=1$ on $B\left(P_{j}, r\right)$, we let $\pi_{n}\left(x^{\prime}, x_{n}\right)=x^{\prime}$ be projection on the first $n-1$ coordinates and define

$$
P\left(\left(f_{j}\right)_{1 \leq j \leq N}\right)(Q)=\sum_{j=1}^{N} \tilde{\eta}_{j}(Q) f_{j}\left(\pi_{n} \circ \varphi_{j}(Q)\right) \quad \text { for } Q \in \partial \Omega .
$$

With these definitions, (1.3) then follows easily from Proposition 1.5. The statement (1.4) may be proved in the same manner. 
The Stokes operator. To introduce the Stokes operator A, let

$$
C_{0, \sigma}^{\infty}(\Omega)=\left\{\varphi \in C_{0}^{\infty}(\Omega): \operatorname{div} \varphi=0\right\}
$$

and $L_{\sigma}^{2}(\Omega)$ be the closure of $C_{0, \sigma}^{\infty}(\Omega)$ in $L^{2}(\Omega)$. We first define a quadratic form $Q$ on $C_{0, \sigma}^{\infty}(\Omega)$ :

$$
Q(u, v)=\int_{\Omega} \nabla u \cdot \nabla v d X=\sum_{j=1}^{n} \int_{\Omega} \nabla u_{j} \cdot \nabla v_{j} d X
$$

We then extend this form to $\mathcal{D}(Q)$, the closure of $C_{0, \sigma}^{\infty}(\Omega)$ in the norm

$$
\|u\|_{Q}=\|u\|_{L^{2}(\Omega)}+\{Q(u, u)\}^{1 / 2}
$$

It is known that $([\mathrm{CF}])$

$$
\mathcal{D}(Q)=\left\{u \in W_{0}^{1,2}(\Omega): \operatorname{div} u=0\right\} .
$$

We now define the Stokes operator $A: \mathcal{D}(A) \rightarrow L_{\sigma}^{2}(\Omega)$ by

$$
\int_{\Omega} A u \cdot \varphi=Q(u, \varphi) \quad \text { for all } \varphi \in C_{0, \sigma}^{\infty}(\Omega)
$$

where the domain of $A, \mathcal{D}(A)$, is the collection of $u$ in $\mathcal{D}(Q)$ such that $v \rightarrow Q(u, v)$ can be extended to a bounded linear functional on $L_{\sigma}^{2}(\Omega)$. It is well known that $A$ gives a self-adjoint operator on $L_{\sigma}^{2}(\Omega)$. Also, it is not hard to see that

$$
\mathcal{D}(A)=\left\{u \in \mathcal{D}(Q):-\Delta u+\nabla \pi \in L_{\sigma}^{2}(\Omega) \text { for some } \pi \in L^{2}(\Omega)\right\}
$$

and

$$
A u=-\Delta u+\nabla \pi, \quad \text { for } u \in \mathcal{D}(A) .
$$

This is the definition of the Stokes operator given in [DW].

Finally, we will follow the standard practice of letting $C$ denote a constant which varies. Throughout this paper $C$ will depend at most on the dimension $n$ and the Lipschitz domain $\Omega$ through the collection of balls used to cover the boundary and the maximum of $\left\|\nabla \psi_{i}\right\|_{\infty}$ where $\psi_{i}$ are the functions whose graphs define $\partial \Omega$. 
2. The Imbedding of $\mathcal{D}(A)$. In this section we will establish the imbedding estimate (0.1) for the domain of $A$.

We will need to consider both the Dirichlet problem with nonzero forcing term $f$ :

$$
\begin{cases}-\Delta u+\nabla \pi=f & \text { in } \Omega, \\ \operatorname{div} u=0 & \text { in } \Omega, \\ u=0 & \text { on } \partial \Omega,\end{cases}
$$

and the problem with inhomogeneous boundary data:

$$
\begin{cases}-\Delta u+\nabla \pi=0 & \text { in } \Omega, \\ \operatorname{div} u=0 & \text { in } \Omega, \\ u=g & \text { on } \partial \Omega .\end{cases}
$$

Obviously, since $\operatorname{div} u=0$ in $\Omega$, the boundary data $g$ should verify the necessary condition

$$
\int_{\partial \Omega}\langle g, N\rangle d \sigma=0
$$

where $N$ denotes the outward unit normal to $\partial \Omega$ and $\langle$,$\rangle the scalar product on$ $\mathbb{R}^{n}$.

The following result is due to Fabes, Kenig and Verchota [FKV]. The estimates for the nontangential maximal functions, the existence and the uniqueness may be found in their paper. The estimates for the solution in Sobolev spaces were announced in that paper, but no proof has appeared. The proof may be obtained by combining the area integral estimates in the appendix of this paper, with the argument given by E. Fabes in $[F]$ to establish the corresponding Sobolev estimates for harmonic functions. We remark that in the first inequality on the top of page 69 of $[\mathrm{F}]$, the integration on $\mathbb{R}^{n-1} \times \mathbb{R}^{n-1}$ should only range over $\{(x, y):|x-y|>t\}$.

Theorem 2.2 (Fabes, Kenig and Verchota [FKV]). Let $\Omega$ be a bounded Lipschitz domain in $\mathbb{R}^{n}, n \geq 3$. Suppose $g \in L^{2}(\partial \Omega)$ and verifies the condition (2.1). Then there exists a solution $u$ to (BVP) which satisfies

$$
\int_{\partial \Omega}\left|(u)^{*}\right|^{2} d \sigma \leq C \int_{\partial \Omega}|g|^{2} d \sigma .
$$

This solution is unique in the class of $u$ satisfying $(u)^{*} \in L^{2}(\partial \Omega)$. In addition, we have the estimate

$$
\|u\|_{W^{1 / 2,2}(\Omega)} \leq C \int_{\partial \Omega}|g|^{2} d \sigma .
$$

Furthermore, if $g \in W^{1,2}(\partial \Omega)$, then we also have

$$
\left\|(\nabla u)^{*}\right\|_{L^{2}(\partial \Omega)}+\|u\|_{W^{3 / 2,2}(\Omega)} \leq C\|g\|_{W^{1,2}(\partial \Omega)} .
$$

We shall also need the Hölder estimates established in [S2, Theorem 0.2, p. 801] for the three-dimensional Lipschitz domains. 
Theorem 2.3 (Z. Shen [S2]). Let $\Omega$ be a bounded Lipschitz domain in $\mathbb{R}^{3}$. There exists $\alpha_{0}>0$ so that if $0<\alpha<\alpha_{0}$, and $g \in C^{\alpha}(\partial \Omega)$ verifies the condition (2.1), then the solution to (DP) lies in $C^{\alpha}(\bar{\Omega})$ and satisfies

$$
\sup _{X \in \Omega} \delta(X)^{1-\alpha}|\nabla u(X)| \leq C\|g\|_{C^{\alpha}(\partial \Omega)}
$$

where $\delta(X)=\operatorname{dist}(X, \partial \Omega)$.

Our estimates for the Stokes operator $A$ will be obtained by interpolating between the estimates of Theorem 2.2 and 2.3. We begin with the following lemma.

Lemma 2.4. Let $\Omega$ be a bounded Lipschitz domain in $\mathbb{R}^{3}$. There exists $\varepsilon>0$ so that if $2 \leq p \leq 3+\varepsilon$, and $g \in W^{1-1 / p, p}(\partial \Omega)$ satisfies the condition (2.1), then the solution $u$ of (BVP) for Stokes equations with boundary data $g$ satisfies

$$
\left(\int_{\Omega}|\nabla u(X)|^{p} d X\right)^{1 / p} \leq C\|g\|_{W^{1-1 / p, p}(\partial \Omega)} .
$$

Proof. Let $\eta$ be a smooth vector field on $\mathbb{R}^{3}$ such that $\langle\eta, N\rangle \geq c_{0}>0$ a.e. on $\partial \Omega$. Recall that $N$ is the outward unit normal to $\partial \Omega$.

For $g \in W^{\alpha, p}(\partial \Omega)$, we define $S g$ by

$$
S g(P)=g(P)-\frac{\int_{\partial \Omega}\langle g, N\rangle d \sigma}{\int_{\partial \Omega}\langle\eta, N\rangle d \sigma} \cdot \eta(P) \quad \text { for } P \in \partial \Omega .
$$

It is easy to see that

$$
\int_{\partial \Omega}\langle S g, N\rangle d \sigma=0
$$

Now let $u$ be the solution of (BVP) with boundary data $S g$. We observe that Theorem 2.2, the area integral estimate in Appendix A (Theorem A.1) and interior estimates imply that

$$
\begin{aligned}
& \left(\int_{\Omega} \delta(X)^{3}\left|\nabla^{2} u(X)\right|^{2} d X\right)^{1 / 2} \leq C\|S g\|_{L^{2}(\partial \Omega)} \leq C\|g\|_{L^{2}(\partial \Omega)} \\
& \left(\int_{\Omega} \delta(X)\left|\nabla^{2} u(X)\right|^{2} d X\right)^{1 / 2} \leq C\|S g\|_{W^{1,2}(\partial \Omega)} \leq C\|g\|_{W^{1,2}(\partial \Omega)}
\end{aligned}
$$


It then follows from the complex interpolation that

$$
\left(\int_{\Omega}\left(\delta(X)^{3 / 2-\alpha}\left|\nabla^{2} u(X)\right|\right)^{2} d X\right)^{1 / 2} \leq C\|g\|_{W^{\alpha, 2}(\partial \Omega)}, \quad 0<\alpha<1 .
$$

where we have used (1.3).

Now, since $\Omega \subset \mathbb{R}^{3}$, we may apply Theorem 2.3 and the interior estimates to obtain

$$
\sup _{X \in \Omega}\left\{\delta(X)^{2-\gamma}\left|\nabla^{2} u(X)\right|\right\} \leq C\|S g\|_{C^{\gamma}(\partial \Omega)} \leq C\|g\|_{C^{\gamma}(\partial \Omega)}
$$

for $0<\gamma<\alpha_{0}$. If we interpolate between (2.5) and (2.6) and use (1.4), we obtain that

$$
\left(\int_{\Omega}\left\{\delta(X)\left|\nabla^{2} u(X)\right|\right\}^{p} d X\right)^{1 / p} \leq C\|g\|_{W^{1-1 / p, p}(\partial \Omega)}
$$

for $2<p<3+\varepsilon$.

To see this, set $\vartheta=2 / p<1$, and choose $\alpha$ and $\gamma$ so that

$$
1=\left(\frac{3}{2}-\alpha\right) \vartheta+(2-\gamma)(1-\vartheta)
$$

Thus,

$$
t=\alpha \vartheta+\gamma(1-\vartheta)=1-\frac{1}{p} .
$$

We use (1.4) to identify the interpolation space

$$
\left[W^{\alpha, 2}(\partial \Omega), C^{\gamma}(\partial \Omega)\right]_{\vartheta}=W^{t, p}(\partial \Omega) .
$$

Note that $2 / p<1$ implies that $p>2$ and the restrictions that $\alpha<1$ and $\gamma<\alpha_{0}$ imply that

$$
\vartheta=\frac{1-\gamma}{\alpha+\frac{1}{2}-\gamma}>\frac{2\left(1-\alpha_{0}\right)}{3-2 \alpha_{0}}
$$

or

$$
p=\frac{2}{\vartheta}<\frac{3-2 \alpha_{0}}{1-\alpha_{0}} \equiv 3+\varepsilon, \quad \varepsilon=\frac{\alpha_{0}}{1-\alpha_{0}}>0 .
$$

Finally, by the Hardy inequality [St, p. 272],

$$
\begin{aligned}
\int_{\Omega}|\nabla u(X)|^{p} d X & \leq C \int_{\Omega}\left|\delta(X) \nabla^{2} u(X)\right|^{p} d X+\sup _{X \in K}|\nabla u(X)|^{p} \\
& \leq C\|g\|_{W^{1-1 / p, p}(\partial \Omega)}^{p},
\end{aligned}
$$

where $K$ is a compact subset of $\Omega$. The proof is complete. 
Let $\Gamma(X)=\left(\Gamma_{i j}(X)\right)_{1 \leq i, j \leq n}$ be the matrix of fundamental solutions and $q(X)=\left(q^{j}(X)\right)_{1 \leq j \leq n}$ be the corresponding pressure vector where

$$
\Gamma_{i j}(X)=\frac{1}{2 \omega_{n}}\left(\frac{1}{(n-2)} \frac{\delta_{i j}}{|X|^{n-2}}+\frac{x_{i} x_{j}}{|X|^{n}}\right), \quad q^{j}(X)=\frac{1}{\omega_{n}} \frac{x_{j}}{|X|^{n}} .
$$

Theorem 2.9. Let $\Omega$ be a bounded Lipschitz domain in $\mathbb{R}^{3}$. Suppose $f \in$ $W^{-1, p}(\Omega)$ where $(3+\varepsilon) /(2+\varepsilon)<p<3+\varepsilon$ and $\varepsilon$ is the same as in Lemma 2.4. Then there exist $u \in W_{0}^{1, p}(\Omega)$ and $\pi \in L^{p}(\Omega)$ so that

$$
\left\{\begin{array}{l}
-\Delta u+\nabla \pi=f \\
\operatorname{div} u=0
\end{array}\right.
$$

in $\Omega$ and

$$
\|\nabla u\|_{L^{p}(\Omega)}+\inf _{c \in \mathbb{R}}\|\pi-c\|_{L^{p}(\Omega)} \leq C\|f\|_{W^{-1, p}(\Omega)} .
$$

The solution $u$ is unique and $\pi$ is unique up to a constant.

Proof. Let $2<p<3+\varepsilon$ and $f \in W^{-1, p}(\Omega)$. We may extend $f$ to lie in $W^{-1, p}\left(\mathbb{R}^{3}\right)$. Let $v=\Gamma * f \in W^{1, p}\left(\mathbb{R}^{3}\right)$. By the trace theorem [Gr, p. 33], $v \in W^{1-1 / p, p}(\partial \Omega)$ and we obtain

$$
\|v\|_{W^{1-1 / p, p}(\partial \Omega)} \leq C\|v\|_{W^{1, p}(\Omega)} \leq C\|f\|_{W^{-1, p}(\Omega)} .
$$

Now let $u=v-w$ where $w$ is the solution of (BVP) for the Stokes equations with boundary data $\left.v\right|_{\partial \Omega}$. Then $u$ satisfies $(2.10)$ and

$$
\begin{aligned}
\|\nabla u\|_{L^{p}(\Omega)} & \leq\|\nabla v\|_{L^{p}(\Omega)}+\|\nabla w\|_{L^{p}(\Omega)} \\
& \leq C\left\{\|f\|_{W^{-1, p}(\Omega)}+\|v\|_{W^{1-1 / p, p}(\partial \Omega)}\right\} \\
& \leq C\|f\|_{W^{-1, p}(\Omega)}
\end{aligned}
$$

by Lemma 2.4 and (2.11).

We may obtain the existence of a solution and the estimates of $u$ for $(3+\varepsilon) /(2+\varepsilon)<p<2$ by duality.

For the pressure term $\pi$, we have

$$
\begin{aligned}
\inf _{c \in \mathbb{R}}\|\pi-c\|_{L^{p}(\Omega)} & \leq\|\nabla \pi\|_{W^{-1, p}(\Omega)} \leq\|\Delta u\|_{W^{-1, p}(\Omega)}+\|f\|_{W^{-1, p}(\Omega)} \\
& \leq C\|\nabla u\|_{L^{p}(\Omega)}+\|f\|_{W^{-1, p}(\Omega)} \leq C\|f\|_{W^{-1, p}(\Omega)} .
\end{aligned}
$$

Finally, the uniqueness for $p \geq 2$ follows by energy estimates. If $p<2$, let $-\Delta u+\nabla \pi=0$ and $u \in W_{0}^{1, p}(\Omega)$. Choose $f \in L^{p^{\prime}}(\Omega)$. We may solve $-\Delta v+\nabla q=f, \operatorname{div} v=0$ in $\Omega, v \in W^{1, p^{\prime}}(\Omega)$, and thus obtain that

$$
\int_{\Omega}\langle f, u\rangle d X=\int_{\Omega} \nabla v \cdot \nabla u d X=0, \quad \text { for any } f \in L^{p^{\prime}}(\Omega),
$$

or that $u=0$. 
Our next result, which is valid for Lipschitz domains in $\mathbb{R}^{n}(n \geq 3)$, gives a sharper estimate for solutions of the Dirichlet problem. It will yield the best embedding of $\mathcal{D}(A)$ in the scale of Sobolev spaces $W^{s, 2}(\Omega)$.

Theorem 2.12. Let $\Omega$ be a bounded Lipschitz domain in $\mathbb{R}^{n}(n \geq 3)$ and $f \in L^{2}(\Omega)$. Suppose that $(u, \pi)$ is the solution of (DP) with data $f$, then for $q_{0}=2 n /(n+1)$, we have the estimate

$$
\|u\|_{W^{3 / 2,2}(\Omega)}+\inf _{c \in \mathbb{R}}\|\pi-c\|_{W^{1 / 2,2}(\Omega)} \leq C\|f\|_{L^{q_{0}(\Omega)}} .
$$

Proof. We construct the solution of (DP) as the sum of the free space solution $(v, \tilde{\pi})=(\Gamma * f, q * f)$ and the solution of the boundary value problem

$$
\begin{cases}-\Delta w+\nabla \psi=0 & \text { in } \Omega, \\ \operatorname{div} w=0 & \text { in } \Omega, \\ w=-v & \text { on } \partial \Omega .\end{cases}
$$

By the Calderón-Zygmund estimates [St], we have

$$
\left\|\nabla^{2} v\right\|_{L^{p}\left(\mathbb{R}^{n}\right)}+\|\nabla \tilde{\pi}\|_{L^{p}\left(\mathbb{R}^{n}\right)} \leq C\|f\|_{L^{p}\left(\mathbb{R}^{n}\right)}
$$

for $1<p<\infty$. This estimate with $p=q_{0}=2 n /(n+1)$ and the Sobolev embedding theorem imply that

$$
\|v\|_{W^{3 / 2,2}(\Omega)}+\|\tilde{\pi}\|_{W^{1 / 2,2}(\Omega)} \leq C\|f\|_{L^{q_{0}}(\Omega)} .
$$

Now consider $(w, \psi)$. We claim that the boundary values of $w,-\left.\Gamma * f\right|_{\partial \Omega}$, satisfy

$$
\|w\|_{W^{1,2}(\partial \Omega)} \leq C\|f\|_{L^{q_{0}}(\Omega)} .
$$

Then the desired estimate,

$$
\|w\|_{W^{3 / 2,2}(\Omega)}+\|\psi\|_{W^{1 / 2,2}(\Omega)} \leq C\|f\|_{L^{q_{0}}(\Omega)},
$$

will follow from Theorem 2.2 .

To establish (2.14), we observe that

$$
\int_{\partial \Omega}|\nabla v|^{2} d \sigma \leq C \int_{\Omega}\left(|\nabla v|^{2}+|\nabla v|\left|\nabla^{2} v\right|\right) d X .
$$

This follows by applying the divergence theorem to $\eta \cdot|\nabla v|^{2}$ where $\eta$ is a smooth vector field on $\mathbb{R}^{n}$ with the property $\langle\eta, N\rangle \geq c_{0}>0$ a.e. on $\partial \Omega$. By (2.13), we have $\left|\nabla^{2} v\right| \in L^{q_{0}}\left(\mathbb{R}^{n}\right)$, while

$$
|\nabla v(X)| \leq C \int_{\Omega} \frac{|f(Y)|}{|X-Y|^{n-1}} d Y
$$

satisfies

$$
\|\nabla v\|_{L^{q_{0}^{\prime}\left(\mathbb{R}^{n}\right)}} \leq C\|f\|_{L^{q_{0}\left(\mathbb{R}^{n}\right)},}, \quad q_{0}^{\prime}=\frac{q_{0}}{q_{0}-1} .
$$

Since $q_{0}^{\prime}>2$, the estimate for $\nabla w$ on $\partial \Omega$ follows from (2.15), (2.16) and (2.13). The estimate for $w$ is easier and we omit the details. 
Theorem 2.17. Suppose $\Omega$ is a bounded Lipschitz domain in $\mathbb{R}^{3}$. Then

$$
\mathcal{D}(A) \subset W_{0}^{1, p}(\Omega) \cap W^{3 / 2,2}(\Omega)
$$

for some $p=p(\Omega)>3$. Moreover, for $u \in \mathcal{D}(A)$,

$$
\|u\|_{W_{0}^{1, p}(\Omega)}+\|u\|_{W^{3 / 2,2}(\Omega)} \leq C\|A u\|_{L^{2}(\Omega)} .
$$

Proof. Let $u \in \mathcal{D}(A)$. Then

$$
\begin{cases}-\Delta u+\nabla \pi=f & \text { in } \Omega, \\ \operatorname{div} u=0 & \text { in } \Omega, \\ u \in W_{0}^{1,2}(\Omega), & \end{cases}
$$

where $f=A u \in L_{\sigma}^{2}(\Omega)$. Since

$$
L^{2}(\Omega) \subset W^{-1, p}(\Omega)
$$

for $p \leq 6$ in $\mathbb{R}^{3}$, the theorem follows immediately from Theorems 2.9 and 2.12 .

3. Some $L^{\infty}$ estimates. In this section we will give the proof of the estimate $(0.2)$. We are only able to prove this estimate in three dimensions and thus throughout this section we will assume that $\Omega$ is a bounded Lipschitz domain in $\mathbb{R}^{3}$.

Theorem 3.1. There exists a constant $C>0$ such that, for $u \in \mathcal{D}(A)$,

$$
\|u\|_{L^{\infty}(\Omega)} \leq C\|\nabla u\|_{L^{2}(\Omega)}^{1 / 2}\|A u\|_{L^{2}(\Omega)}^{1 / 2} .
$$

By the definition of $A$ and a limiting argument,

$$
\int_{\Omega} A u \cdot u d X=\int_{\Omega} \nabla u \cdot \nabla u d X
$$

It then follows from the Cauchy inequality that

$$
\|\nabla u\|_{L^{2}(\Omega)}^{2} \leq\|A u\|_{L^{2}(\Omega)}\|u\|_{L^{2}(\Omega)} .
$$

Thus, we have: 
Corollary 3.2. There exists a constant $C>0$ such that, for $u \in \mathcal{D}(A)$,

$$
\|u\|_{L^{\infty}(\Omega)} \leq C\|u\|_{L^{2}(\Omega)}^{1 / 4}\|A u\|_{L^{2}(\Omega)}^{3 / 4} .
$$

As in the case of smooth domains, $A^{-1}: L_{\sigma}^{2}(\Omega) \rightarrow L_{\sigma}^{2}(\Omega)$ is a compact operator. Hence, there exists a sequence of positive numbers $\left\{\lambda_{k}\right\}$ and an orthonormal basis $\left\{\omega_{k}\right\}$ of $L_{\sigma}^{2}(\Omega)$ such that $A \omega_{k}=\lambda_{k} \omega_{k}, \omega_{k} \in \mathcal{D}(A)$ and $\lim _{k \rightarrow \infty} \lambda_{k}=\infty$.

Corollary 3.3. Let $\Omega$ be a bounded Lipschitz domain in $\mathbb{R}^{3}$. Then there exists $c>0$ such that

$$
\lambda_{k} \geq c\left(\frac{k}{|\Omega|}\right)^{2 / 3}
$$

With Corollary 3.2, the proof is exactly the same as in the smooth case. See [CF, p. 38-39].

To prove Theorem 3.1, we start with a reverse Hölder inequality.

Lemma 3.4. Let $X_{0} \in \bar{\Omega}, R>0$ be small, and $D\left(X_{0}, R\right)=B\left(X_{0}, R\right) \cap \Omega$. Assume $(u, \pi)$ satisfies the Stokes equations

$$
\left\{\begin{array}{l}
-\Delta u+\nabla \pi=0 \\
\operatorname{div} u=0
\end{array}\right.
$$

in $D\left(X_{0}, 8 R\right)$ and $u=0$ on $\partial \Omega$. Then

$$
\left(\frac{1}{R^{3}} \int_{D\left(X_{0}, R\right)}|\nabla u|^{p_{0}} d X\right)^{1 / p_{0}} \leq C\left(\frac{1}{R^{3}} \int_{D\left(X_{0}, 4 R\right)}|\nabla u|^{2} d X\right)^{1 / 2}
$$

where $p_{0}>2$ depends only on $\Omega$.

Proof. We begin with a Caccioppoli type inequality for the Stokes equations (see [S2, Lemma 1.5, p. 804]). We consider two cases. If $B\left(X_{0}, 2 R\right) \cap \partial \Omega \neq \varnothing$, we have

$$
\int_{D\left(X_{0}, R\right)}|\nabla u|^{2} d X \leq \frac{C}{R^{2}} \int_{D\left(X_{0}, 3 R\right)}|u|^{2} d X .
$$

On the other hand, if $B\left(X_{0}, 2 R\right) \subset \Omega$, we get

$$
\int_{D\left(X_{0}, R\right)}|\nabla u|^{2} d X \leq \frac{C}{R^{2}} \int_{D\left(X_{0}, 3 R\right)}\left|u-u_{R}\right|^{2} d X
$$

where

$$
u_{R}=\frac{1}{\left|B\left(X_{0}, 2 R\right)\right|} \int_{B\left(X_{0}, 2 R\right)} u(X) d X
$$


In both cases, by Sobolev-Poincaré inequality, we obtain

$$
\begin{aligned}
& \left(\frac{1}{\left|D\left(X_{0}, R\right)\right|} \int_{D\left(X_{0}, R\right)}|\nabla u|^{2} d X\right)^{1 / 2} \\
\leq & C\left(\frac{1}{\left|D\left(X_{0}, 3 R\right)\right|} \int_{D\left(X_{0}, 3 R\right)}|\nabla u|^{6 / 5} d X\right)^{5 / 6} .
\end{aligned}
$$

The lemma then follows from the usual reverse Hölder inequality (e.g., see [Gi, Proposition 1.1, p. 122]).

We now give the:

Proof of Theorem 3.1. Let $u \in \mathcal{D}(A)$ and $f=A u \in L_{\sigma}^{2}(\Omega)$. Fix $X_{0} \in \Omega$. Let $D_{t}=B\left(X_{0}, t\right) \cap \Omega$ where $t \in(0,1)$ is to be chosen later.

Let $f_{1}=f$ in $D_{t}$ and 0 otherwise. Let $f_{2}=f-f_{1}$. We use $\left(u_{i}, \pi_{i}\right), i=1,2$, to denote solutions of (DP) for the Stokes equations with data $f_{i}$. By uniqueness, $u=u_{1}+u_{2}$.

First we estimate $u_{1}\left(X_{0}\right)$. We claim that

$$
\left|u_{1}\left(X_{0}\right)\right| \leq C t^{1 / 2}\|f\|_{L^{2}(\Omega)} .
$$

Indeed, for any $X \in D_{t}$, by the imbedding theorem of Morrey [GT, Theorem 7.17, p. 163],

$$
\left|u_{1}(X)-u_{1}\left(X_{0}\right)\right| \leq C t^{\gamma}\left\|\nabla u_{1}\right\|_{L^{p}(\Omega)},
$$

where $p>3$ and $\gamma=1-3 / p$. It then follows from Theorem 2.9 that

$$
\left|u_{1}(X)-u_{1}\left(X_{0}\right)\right| \leq C t^{\gamma}\left\|f_{1}\right\|_{W^{-1, p}(\Omega)} \leq C t^{\gamma}\left\|f_{1}\right\|_{L^{q}(\Omega)} \leq t^{1 / 2}\|f\|_{L^{2}(\Omega)},
$$

where $1 / q=1 / p+1 / 3$ and we also used the Sobolev imbedding theorem [GT, Theorem 7.26, p. 171] and Hölder inequality (we also assume that $p<6$ ).

Now we integrate the inequality above in $X$ over $D_{t}$. This, together with the Sobolev imbedding, gives

$$
\begin{aligned}
\left|u_{1}\left(X_{0}\right)\right| & \leq\left(\frac{1}{\left|D_{t}\right|} \int_{D_{t}}\left|u_{1}\right|^{6} d X\right)^{1 / 6}+C t^{1 / 2}\|f\|_{L^{2}(\Omega)} \\
& \leq C t^{-1 / 2}\left\|\nabla u_{1}\right\|_{L^{2}(\Omega)}+C t^{1 / 2}\|f\|_{L^{2}(\Omega)} \\
& \leq C t^{-1 / 2}\left\|f_{1}\right\|_{W^{-1,2}(\Omega)}+C t^{1 / 2}\|f\|_{L^{2}(\Omega)} \\
& \leq C t^{-1 / 2}\left\|f_{1}\right\|_{L^{6 / 5}(\Omega)}+C t^{1 / 2}\|f\|_{L^{2}(\Omega)} \\
& \leq C t^{1 / 2}\|f\|_{L^{2}(\Omega)} .
\end{aligned}
$$


To estimate $u_{2}\left(X_{0}\right)$, let $s \in[t / 8, t / 4]$ and $X \in D_{s}$. Note that $-\Delta u_{2}+\nabla \pi_{2}=$ 0 and $\operatorname{div} u_{2}=0$ in $D_{s}$. We may apply Theorem 2.3 on $D_{s}$ to obtain

$$
\begin{aligned}
\left|u_{2}(X)-u_{2}\left(X_{0}\right)\right| & \leq C t^{\alpha} \sup _{\substack{P_{1}, P_{2} \in \partial D_{s} \\
P_{1} \neq P_{2}}} \frac{\left|u_{2}\left(P_{1}\right)-u_{2}\left(P_{2}\right)\right|}{\left|P_{1}-P_{2}\right|^{\alpha}} \\
& \leq C t^{\alpha}\left(\int_{\partial D_{s}}\left|\nabla u_{2}\right|^{p_{0}} d \sigma\right)^{1 / p_{0}},
\end{aligned}
$$

where $p_{0}=2 /(1-\alpha)>2$ and we have used the Sobolev imbedding on the set $\partial D_{s}$. By integration in $s$ over $[t / 8, t / 4]$, we get

$$
\begin{aligned}
\left|u_{2}(X)-u_{2}\left(X_{0}\right)\right| & \leq C t^{\alpha-1 / p_{0}}\left(\int_{D_{t / 4}}\left|\nabla u_{2}\right|^{p_{0}} d X\right)^{1 / p_{0}} \\
& \leq C t\left(\frac{1}{\left|D_{t / 4}\right|} \int_{D_{t / 4}}\left|\nabla u_{2}\right|^{p_{0}} d X\right)^{1 / p_{0}} \\
& \leq C t\left(\frac{1}{\left|D_{t}\right|} \int_{D_{t}}\left|\nabla u_{2}\right|^{2} d X\right)^{1 / 2} \\
& \leq C t\left\{\left(\frac{1}{\left|D_{t}\right|} \int_{D_{t}}|\nabla u|^{2} d X\right)^{1 / 2}+\left(\frac{1}{\left|D_{t}\right|} \int_{D_{t}}\left|\nabla u_{1}\right|^{2} d X\right)^{1 / 2}\right\} \\
& \leq C t^{-1 / 2}\|\nabla u\|_{L^{2}(\Omega)}+C t^{-1 / 2}\left\|\nabla u_{1}\right\|_{L^{2}(\Omega)} \\
& \leq C t^{-1 / 2}\|\nabla u\|_{L^{2}(\Omega)}+C t^{1 / 2}\|f\|_{L^{2}(\Omega)},
\end{aligned}
$$

where we used the reverse Hölder inequality (Lemma 3.4) in the third inequality.

Thus,

$$
\begin{aligned}
\left|u_{2}\left(X_{0}\right)\right| & \leq\left(\frac{1}{\left|D_{t}\right|} \int_{D_{t}}\left|u_{2}\right|^{6} d X\right)^{1 / 6}+C t^{-1 / 2}\|\nabla u\|_{L^{2}(\Omega)}+C t^{1 / 2}\|f\|_{L^{2}(\Omega)} \\
& \leq C t^{-1 / 2}\left\|u_{2}\right\|_{L^{6}(\Omega)}+C t^{-1 / 2}\|\nabla u\|_{L^{2}(\Omega)}+C t^{1 / 2}\|f\|_{L^{2}(\Omega)} \\
& \leq C t^{-1 / 2}\left\|\nabla u_{2}\right\|_{L^{2}(\Omega)}+C t^{-1 / 2}\|\nabla u\|_{L^{2}(\Omega)}+C t^{1 / 2}\|f\|_{L^{2}(\Omega)} \\
& \leq C t^{-1 / 2}\|\nabla u\|_{L^{2}(\Omega)}+C t^{-1 / 2}\left\|\nabla u_{1}\right\|_{L^{2}(\Omega)}+C t^{1 / 2}\|f\|_{L^{2}(\Omega)} \\
& \leq C t^{-1 / 2}\|\nabla u\|_{L^{2}(\Omega)}+C t^{1 / 2}\|f\|_{L^{2}(\Omega)} .
\end{aligned}
$$

To summarize, we have proved that

$$
\left|u\left(X_{0}\right)\right| \leq C t^{-1 / 2}\|\nabla u\|_{L^{2}(\Omega)}+C t^{1 / 2}\|f\|_{L^{2}(\Omega)}
$$


for any $t \in\left(0, c_{0}\right)$, where $c_{0}$ depends only on $\Omega$.

Finally, by the energy estimate, $\|\nabla u\|_{L^{2}(\Omega)} \leq C_{0}\|f\|_{L^{2}(\Omega)}$, so we may choose

$$
t=\frac{c_{0}\|\nabla u\|_{L^{2}(\Omega)}}{2 C_{0}\|f\|_{L^{2}(\Omega)}}<c_{0}
$$

to obtain

$$
\left|u\left(X_{0}\right)\right| \leq C\|\nabla u\|_{L^{2}(\Omega)}^{1 / 2}\|A u\|_{L^{2}(\Omega)}^{1 / 2} .
$$

The proof is finished.

Appendix A. In this appendix, we present a simple proof of the area integral estimates for solutions of Stokes equations.

Theorem A.1. Let $\Omega$ be a bounded Lipschitz domain in $\mathbb{R}^{n}, n \geq 3$. Suppose $\Delta u=\nabla \pi, \operatorname{div} u=0$ in $\Omega$, and $(u)^{*} \in L^{2}(\partial \Omega)$. Then

$$
\int_{\Omega}|\nabla u(X)|^{2} \delta(X) d X \leq C \int_{\partial \Omega}|u|^{2} d \sigma
$$

and

$$
\int_{\partial \Omega}|u|^{2} d \sigma \leq C\left\{\int_{\Omega}|\nabla u(X)|^{2} \delta(X) d X+\int_{\Omega}|u(X)|^{2} d X\right\},
$$

where $\delta(X)=\operatorname{dist}(X, \partial \Omega)$.

Theorem A.1 is due to E. Fabes, C. Kenig and G. Verchota (unpublished). The proof given here is based on an idea of B. Dahlberg, C. Kenig, J. Pipher and G. Verchota [DKPV] developed for elliptic systems, and some observations on the pressure term $\pi$.

The following lemma is due to C. Kenig and E. Stein.

Lemma A.2. Suppose $\psi: \mathbb{R}^{n-1} \rightarrow \mathbb{R}$ is a Lipschitz function. Let $\vartheta \in$ $C_{0}^{\infty}\left(\mathbb{R}^{n-1}\right)$ be radial, and $\vartheta \geq 0, \int \vartheta d x=1$. Then, if $\lambda \geq C\left(n,\|\nabla \psi\|_{L^{\infty}}\right)$,

$$
(x, t) \rightarrow(x, y)=(x, \varphi(x, t))
$$

is a bi-Lipschitz map from $\mathbb{R}_{+}^{n}$ to $D=\{(x, y): y>\psi(x)\}$ where $x \in \mathbb{R}^{n-1}, t, y \in$ $\mathbb{R}$ and

$$
\varphi(x, t)=\lambda t+\left(\vartheta_{t} * \psi\right)(x), \quad \vartheta_{t}(x)=\frac{1}{t^{n-1}} \vartheta\left(\frac{x}{t}\right) .
$$

Moreover, $\left|\nabla^{2} \varphi(x, t)\right|^{2} t d x d t$ is a Carleson measure on $\mathbb{R}_{+}^{n}$. 
Lemma A.3. Let $D=\left\{(x, y) \in \mathbb{R}^{n}: y>\psi(x)\right\}$ where $\psi: \mathbb{R}^{n-1} \rightarrow \mathbb{R}$ is a Lipschitz function. Suppose that

$$
\left\{\begin{array}{l}
-\Delta u+\nabla \pi=f, \\
\operatorname{div} u=g,
\end{array}\right.
$$

in D. Also assume that $u, \pi$ have compact supports and $(u)^{*} \in L^{2}(\partial D)$. Then

$$
\begin{aligned}
\int_{D}|\nabla u|^{2} \delta(X) d X \leq C\left\{\int_{\partial D}\left|(u)^{*}\right|^{2} d \sigma\right. & +\int_{D}|\pi|^{2} \delta(X) d X+\int_{D}|\nabla \pi|^{2} \delta^{3}(X) d X \\
& \left.+\int_{D}|\Delta g+\operatorname{div} f||u| \delta^{2}(X) d X+\int_{D}|f||u| \delta(X) d X\right\}
\end{aligned}
$$

and

$$
\begin{array}{rl}
\int_{\partial D}|u|^{2} & d \sigma \leq \varepsilon \int_{\partial D}\left|(u)^{*}\right|^{2} d \sigma+C_{\varepsilon}\left\{\int_{D}|\nabla u|^{2} \delta(X) d X+\int_{D}|\pi|^{2} \delta(X) d X\right. \\
& \left.+\int_{D}|\nabla \pi|^{2} \delta^{3}(X) d X+\int_{D}|\Delta g+\operatorname{div} f||u| \delta^{2}(X) d X+\int_{D}|f||u| \delta(X) d X\right\}
\end{array}
$$

for any $\varepsilon>0$.

Proof. Let $\rho: \mathbb{R}_{+}^{n} \rightarrow D$ be defined by

$$
\rho(x, t)=(x, y)=(x, \varphi(x, t)),
$$

where $\varphi(x, t)=\lambda t+\vartheta_{t} * \psi(x)$ is given in Lemma A.2.

Let $v=u \circ \rho, q=\pi \circ \rho$ be defined on $\mathbb{R}_{+}^{n}$. Note that $v$ and $q$ have compact supports in $\mathbb{R}_{+}^{n}$ and satisfy

$$
\left\{\begin{array}{l}
\frac{\partial v}{\partial t}=\frac{\partial u}{\partial y} \circ \rho \cdot \frac{\partial \varphi}{\partial t} \\
\frac{\partial v}{\partial x_{i}}=\frac{\partial u}{\partial x_{i}} \circ \rho+\frac{\partial u}{\partial y} \circ \rho \cdot \frac{\partial \varphi}{\partial x_{i}}, \quad i=1,2, \ldots, n-1 .
\end{array}\right.
$$

It follows from integration by parts that

$$
\text { (A.5) } \begin{aligned}
& \int_{R^{n-1}}|u \circ \rho|^{2} d x=\int_{R^{n-1}}|v|^{2} d x=-2 \iint_{\mathbb{R}_{+}^{n}} \frac{\partial v}{\partial t} \cdot v d x d t \\
= & 2 \iint_{\mathbb{R}_{+}^{n}} t\left|\frac{\partial v}{\partial t}\right|^{2} d x d t+2 \iint_{\mathbb{R}_{+}^{n}} t \frac{\partial^{2} v}{\partial t^{2}} \cdot v d x d t \\
= & 2 \iint_{\mathbb{R}_{+}^{n}} t\left|\frac{\partial \varphi}{\partial t}\right|^{2}\left|\frac{\partial u}{\partial y} \circ \rho\right|^{2} d x d t+2 \iint_{\mathbb{R}_{+}^{n}} t\left|\frac{\partial \varphi}{\partial t}\right|^{2} \frac{\partial^{2} u}{\partial y^{2}} \circ \rho \cdot u \circ \rho d x d t+G,
\end{aligned}
$$


where $G$ denotes terms which are bounded in absolute value by

$$
\begin{aligned}
& C \iint_{\mathbb{R}_{+}^{n}} t\left|\nabla^{2} \varphi\right||\nabla u \circ \rho||u \circ \rho| d x d t \\
& \quad+C \iint_{\mathbb{R}_{+}^{n}} t\left|\nabla^{2} \varphi\right||\pi \circ \rho||u \circ \rho| d x d t \\
& \quad+C \iint_{\mathbb{R}_{+}^{n}} t^{2}\left|\nabla^{2} \varphi\right||\nabla \pi \circ \rho||u \circ \rho| d x d t \\
& \quad+C \iint_{\mathbb{R}_{+}^{n}} t|\pi \circ \rho||\nabla u \circ \rho| d x d t \\
& \quad+C \iint_{\mathbb{R}_{+}^{n}} t^{2}|\nabla \pi \circ \rho||\nabla u \circ \rho| d x d t .
\end{aligned}
$$

By the Stokes equations $\Delta u=\nabla \pi-f$, we have

$$
\begin{aligned}
\frac{\partial^{2} u}{\partial y^{2}} \circ \rho= & -\frac{\partial^{2} u}{\partial x_{i}^{2}} \circ \rho+\nabla \pi \circ \rho-f \circ \rho \\
= & -\frac{\partial}{\partial x_{i}}\left\{\frac{\partial u}{\partial x_{i}} \circ \rho\right\}+\frac{\partial^{2} u}{\partial y \partial x_{i}} \circ \rho \cdot \frac{\partial \varphi}{\partial x_{i}}+\nabla \pi \circ \rho-f \circ \rho \\
= & -\frac{\partial}{\partial x_{i}}\left\{\frac{\partial u}{\partial x_{i}} \circ \rho\right\}+\frac{\partial}{\partial x_{i}}\left\{\frac{\partial u}{\partial y} \circ \rho\right\} \cdot \frac{\partial \varphi}{\partial x_{i}} \\
& -\frac{\partial^{2} u}{\partial y^{2}} \circ \rho \cdot\left|\nabla_{x} \varphi\right|^{2}+\nabla \pi \circ \rho-f \circ \rho,
\end{aligned}
$$

where the repeated index $i$ is summed over $\{1,2, \ldots, n-1\}$ and

$$
\nabla_{x}=\left(\frac{\partial}{\partial x_{1}}, \ldots, \partial \partial x_{n-1}\right)
$$

It follows that

$$
\frac{\partial^{2} u}{\partial y^{2}} \circ \rho=\frac{1}{1+\left|\nabla_{x} \varphi\right|^{2}}\left\{-\frac{\partial}{\partial x_{i}}\left\{\frac{\partial u}{\partial x_{i}} \circ \rho\right\}+\frac{\partial}{\partial x_{i}}\left\{\frac{\partial u}{\partial y} \circ \rho\right\} \cdot \frac{\partial \varphi}{\partial x_{i}}+\nabla \pi \circ \rho-f \circ \rho\right\} .
$$


Thus, we may use integration by parts to obtain

$$
\begin{aligned}
& \iint_{\mathbb{R}_{+}^{n}} t\left|\frac{\partial \varphi}{\partial t}\right|^{2} \frac{\partial^{2} u}{\partial y^{2}} \circ \rho \cdot u \circ \rho d x d t \\
& =\iint_{\mathbb{R}_{+}^{n}} \frac{t\left(\frac{\partial \varphi}{\partial t}\right)^{2}}{1+\left|\nabla_{x} \varphi\right|^{2}} \cdot \frac{\partial u}{\partial x_{i}} \circ \rho \cdot \frac{\partial}{\partial x_{i}}\{u \circ \rho\} d x d t \\
& -\iint_{\mathbb{R}_{+}^{n}} \frac{t\left(\frac{\partial \varphi}{\partial t}\right)^{2} \frac{\partial \varphi}{\partial x_{i}}}{1+\left|\nabla_{x} \varphi\right|^{2}} \cdot \frac{\partial u}{\partial y} \circ \rho \cdot \frac{\partial}{\partial x_{i}}\{u \circ \rho\} d x d t \\
& +\iint_{\mathbb{R}_{+}^{n}} \frac{t\left(\frac{\partial \varphi}{\partial t}\right)^{2}}{1+\left|\nabla_{x} \varphi\right|^{2}} \cdot\{\nabla \pi \circ \rho-f \circ \rho\} \cdot u \circ \rho d x d t+G \\
& =\iint_{\mathbb{R}_{+}^{n}} \frac{t\left(\frac{\partial \varphi}{\partial t}\right)^{2}}{1+\left|\nabla_{x} \varphi\right|^{2}} \cdot\left|\frac{\partial u}{\partial x_{i}} \circ \rho\right|^{2} d x d t \\
& -\iint_{\mathbb{R}_{+}^{n}} \frac{t\left(\frac{\partial \varphi}{\partial t}\right)^{2}\left|\nabla_{x} \varphi\right|^{2}}{1+\left|\nabla_{x} \varphi\right|^{2}} \cdot\left|\frac{\partial u}{\partial y} \circ \rho\right|^{2} d x d t \\
& +\iint_{\mathbb{R}_{+}^{n}} \frac{t\left(\frac{\partial \varphi}{\partial t}\right)^{2}}{1+\left|\nabla_{x} \varphi\right|^{2}} \cdot\{\nabla \pi \circ \rho-f \circ \rho\} \cdot u \circ \rho d x d t+G .
\end{aligned}
$$

This, together with (A.5), implies that

$$
\begin{aligned}
\int_{\mathbb{R}^{n-1}}|u \circ \rho|^{2} d x & =2 \iint_{\mathbb{R}_{+}^{n}} \frac{t\left(\frac{\partial \varphi}{\partial t}\right)^{2}}{1+\left|\nabla_{x} \varphi\right|^{2}} \cdot|\nabla u \circ \rho|^{2} d x d t \\
& +2 \iint_{\mathbb{R}_{+}^{n}} \frac{t\left(\frac{\partial \varphi}{\partial t}\right)^{2}}{1+\left|\nabla_{x} \varphi\right|^{2}} \cdot\{\nabla \pi \circ \rho-f \circ \rho\} \cdot u \circ \rho d x d t+G .
\end{aligned}
$$

It remains to estimate

$$
\iint_{\mathbb{R}_{+}^{n}} \frac{t\left(\frac{\partial \varphi}{\partial t}\right)^{2}}{1+\left|\nabla_{x} \varphi\right|^{2}} \nabla \pi \circ \rho \cdot u \circ \rho d x d t .
$$


To this end, we let $u=\left(u_{1}, u_{2}, \ldots, u_{n}\right)$ and note that

$$
\begin{aligned}
\nabla \pi \circ \rho \cdot u \circ \rho & =\frac{\partial \pi}{\partial x_{i}} \circ \rho \cdot u_{i} \circ \rho+\frac{\partial \pi}{\partial y} \circ \rho \cdot u_{n} \circ \rho \\
& =\frac{\partial}{\partial x_{i}}\{\pi \circ \rho\} \cdot u_{i} \circ \rho+\frac{\partial \pi}{\partial y} \circ \rho \cdot\left\{u_{n} \circ \rho-u_{i} \circ \rho \frac{\partial \varphi}{\partial x_{i}}\right\} .
\end{aligned}
$$

Thus, the integration by parts yields

$$
\begin{aligned}
& 2 \iint_{\mathbb{R}_{+}^{n}} \frac{t\left(\frac{\partial \varphi}{\partial t}\right)^{2}}{1+\left|\nabla_{x} \varphi\right|^{2}} \nabla \pi \circ \rho \cdot u \circ \rho d x d t \\
= & 2 \iint_{\mathbb{R}_{+}^{n}} \frac{t\left(\frac{\partial \varphi}{\partial t}\right)^{2}}{1+\left|\nabla_{x} \varphi\right|^{2}} \frac{\partial \pi}{\partial y} \circ \rho \cdot\left\{u_{n} \circ \rho-u_{i} \circ \rho \frac{\partial \varphi}{\partial x_{i}}\right\} d x d t+G .
\end{aligned}
$$

Now, we write $2 t=\partial t^{2} / \partial t$. Using integration by parts, we obtain

$$
\begin{aligned}
& 2 \iint_{\mathbb{R}_{+}^{n}} \frac{t\left(\frac{\partial \varphi}{\partial t}\right)^{2}}{1+\left|\nabla_{x} \varphi\right|^{2}} \cdot \frac{\partial \pi}{\partial y} \circ \rho \cdot\left\{u_{n} \circ \rho-u_{i} \circ \rho \frac{\partial \varphi}{\partial x_{i}}\right\} d x d t \\
= & -\iint_{\mathbb{R}_{+}^{n}} \frac{t^{2}\left(\frac{\partial \varphi}{\partial t}\right)^{3}}{1+\left|\nabla_{x} \varphi\right|^{2}} \cdot \frac{\partial^{2} \pi}{\partial y^{2}} \circ \rho \cdot\left\{u_{n} \circ \rho-u_{i} \circ \rho \frac{\partial \varphi}{\partial x_{i}}\right\} d x d t+G .
\end{aligned}
$$

Since $\Delta u=\nabla \pi-f$, we have $\Delta \pi=\Delta(\operatorname{div} u)+\operatorname{div} f=\Delta g+\operatorname{div} f$. Thus,

$$
\begin{aligned}
\frac{\partial^{2} \pi}{\partial y^{2}} \circ \rho= & -\frac{\partial^{2} \pi}{\partial x_{i}^{2}} \circ \rho+\Delta g \circ \rho+(\operatorname{div} f) \circ \rho \\
=-\frac{\partial}{\partial x_{i}} & \left\{\frac{\partial \pi}{\partial x_{i}} \circ \rho\right\}+\frac{\partial}{\partial x_{i}}\left\{\frac{\partial \pi}{\partial y} \circ \rho\right\} \cdot \frac{\partial \varphi}{\partial x_{i}}-\frac{\partial^{2} \pi}{\partial y^{2}} \circ \rho\left|\nabla_{x} \varphi\right|^{2} \\
& \quad+\Delta g \circ \rho+(\operatorname{div} f) \circ \rho .
\end{aligned}
$$

It follows that

$$
\frac{\partial^{2} \pi}{\partial y^{2}} \circ \rho=\frac{1}{1+\left|\nabla_{x} \varphi\right|^{2}}\left\{-\frac{\partial}{\partial x_{i}}\left\{\frac{\partial \pi}{\partial x_{i}} \circ \rho\right\}+\frac{\partial}{\partial x_{i}}\left\{\frac{\partial \pi}{\partial y} \circ \rho\right\} \cdot \frac{\partial \varphi}{\partial x_{i}}+\Delta g \circ \rho+(\operatorname{div} f) \circ \rho\right\} .
$$


Hence, the integration by parts again yields

$$
\begin{aligned}
& 2 \iint_{\mathbb{R}_{+}^{n}} \frac{t\left(\frac{\partial \varphi}{\partial t}\right)^{2}}{1+\left|\nabla_{x} \varphi\right|^{2}} \cdot \frac{\partial \pi}{\partial y} \circ \rho \cdot\left\{u_{n} \circ \rho-u_{i} \circ \rho \frac{\partial \varphi}{\partial x_{i}}\right\} d x d t \\
= & -\iint_{\mathbb{R}_{+}^{n}} \frac{t^{2}\left(\frac{\partial \varphi}{\partial t}\right)^{3}}{\left(1+\left|\nabla_{x} \varphi\right|^{2}\right)^{2}}\{\Delta g+\operatorname{div} f\} \circ \rho \cdot\left\{u_{n} \circ \rho-u_{i} \circ \rho \frac{\partial \varphi}{\partial x_{i}}\right\} d x d t+G .
\end{aligned}
$$

Thus, putting together (A.7), (A.8) and the estimate above, we have proved that

$$
\begin{aligned}
& \int_{\mathbb{R}^{n-1}}|u \circ \rho|^{2} d x \\
&=2 \iint_{\mathbb{R}_{+}^{n}} \frac{t\left(\frac{\partial \varphi}{\partial t}\right)^{2}}{1+\left|\nabla_{x} \varphi\right|^{2}}|\nabla u \circ \rho|^{2} d x d t \\
&-\iint_{\mathbb{R}_{+}^{n}} \frac{t^{2}\left(\frac{\partial \varphi}{\partial t}\right)^{3}}{\left(1+\left|\nabla_{x} \varphi\right|^{2}\right)^{2}}\{\Delta g+\operatorname{div} f\} \circ \rho \cdot\left\{u_{n} \circ \rho-u_{i} \circ \rho \frac{\partial \varphi}{\partial x_{i}}\right\} d x d t \\
&-2 \iint_{\mathbb{R}_{+}^{n}} \frac{t\left(\frac{\partial \varphi}{\partial t}\right)^{2}}{1+\left|\nabla_{x} \varphi\right|^{2}} f \circ \rho \cdot u \circ \rho d x d t+G .
\end{aligned}
$$

This implies that

$$
\begin{gathered}
\int_{\partial D}|u|^{2} d \sigma \leq C \int_{D}|\nabla u|^{2} \delta(X) d X+C \int_{D}|\Delta g+\operatorname{div} f||u| \delta^{2}(X) d X \\
+C \int_{D}|f||u| \delta(X) d X+|G|
\end{gathered}
$$

and

$$
\begin{gathered}
\int_{D}|\nabla u|^{2} \delta(X) d X \leq C \int_{\partial D}|u|^{2} d \sigma+C \int_{D}|\Delta g+\operatorname{div} f||u| \delta^{2}(X) d X \\
+C \int_{D}|f||u| \delta(X) d X+|G| .
\end{gathered}
$$


Since $\left|\nabla^{2} \varphi\right|^{2} t d x d t$ is a Carleson measure, by the Cauchy-Schwartz inequality and (A.6), we have

$$
\begin{aligned}
|G| \leq C\left(\int_{D}|\nabla u|^{2} \delta(X) d X\right)^{1 / 2}\left(\int_{\partial D}\left|(u)^{*}\right|^{2} d \sigma\right)^{1 / 2} & \\
+ & C\left(\int_{D}|\pi|^{2} \delta(X) d X\right)^{1 / 2}\left(\int_{\partial D}\left|(u)^{*}\right|^{2} d \sigma\right)^{1 / 2} \\
& +C\left(\int_{D}|\nabla \pi|^{2} \delta^{3}(X) d X\right)^{1 / 2}\left(\int_{\partial D}\left|(u)^{*}\right|^{2} d \sigma\right)^{1 / 2} \\
& +C\left(\int_{D}|\pi|^{2} \delta(X) d X\right)^{1 / 2}\left(\int_{D}|\nabla u|^{2} \delta(X) d X\right)^{1 / 2} \\
& +C\left(\int_{D}|\nabla \pi|^{2} \delta^{3}(X) d X\right)^{1 / 2}\left(\int_{D}|\nabla u|^{2} \delta(X) d X\right)^{1 / 2} .
\end{aligned}
$$

The lemma now follows easily from the Cauchy inequality.

Lemma A.9. Let $\Omega$ be a bounded Lipschitz domain in $\mathbb{R}^{n}, n \geq 3$. Suppose that $\Delta u=\nabla \pi, \operatorname{div} u=0$ in $\Omega$, and $(u)^{*} \in L^{2}(\partial \Omega)$. Then there exists a function $\tilde{\pi}$ such that $\tilde{\pi}=\pi+c$ and

$$
\int_{\Omega}|\tilde{\pi}(X)|^{2} \delta(X) d X+\int_{\Omega}|\nabla \tilde{\pi}(X)|^{2} \delta^{3}(X) d X \leq C \int_{\partial \Omega}|u|^{2} d \sigma .
$$

Proof. By the $L^{2}$ estimates in $[\mathrm{FKV}], u$ can be represented in terms of a double layer potential:

$$
u_{i}(X)=\int_{\partial \Omega}\left\{\frac{\partial}{\partial y_{k}}\left\{\Gamma_{i j}(X-Y)\right\} N_{k}(Y)-q^{i}(X-Y) N_{j}(Y)\right\} h_{j}(Y) d \sigma(Y),
$$

where $\|h\|_{L^{2}(\partial \Omega)} \leq C\|u\|_{L^{2}(\partial \Omega)},\left(\Gamma_{i j}(X)\right)$ is the matrix of fundamental solutions and $\left(q^{i}(X)\right)$ is the corresponding pressure vector given in (2.8).

Note that

$$
\Delta \Gamma_{i j}(X)=\frac{1}{2 \omega_{n}} \Delta\left\{\frac{x_{i} x_{j}}{|X|^{n}}\right\}=\frac{1}{\omega_{n}} \nabla\left(\frac{x_{i}}{|X|^{n}}\right) \cdot \nabla x_{j}=\frac{1}{\omega_{n}} \frac{\partial}{\partial x_{i}}\left\{\frac{x_{j}}{|X|^{n}}\right\} .
$$

Thus,

$$
\begin{aligned}
\Delta u_{i}(X) & =\Delta \int_{\partial \Omega} \frac{\partial}{\partial y_{k}}\left\{\Gamma_{i j}(X-Y)\right\} N_{k}(Y) h_{j}(Y) d \sigma(Y) \\
& =-\frac{\partial}{\partial x_{i}} \frac{\partial}{\partial x_{k}} \int_{\partial \Omega} \frac{x_{j}-y_{j}}{\omega_{n}|X-Y|^{n}} N_{k}(Y) h_{j}(Y) d \sigma(Y)=\frac{\partial \tilde{\pi}}{\partial x_{i}}
\end{aligned}
$$


where

$\tilde{\pi}(X)=-\frac{\partial w_{k}(X)}{\partial x_{k}} \quad$ and $\quad w_{k}(X)=\int_{p o} \frac{x_{j}-y_{j}}{\omega_{n}|X-Y|^{n}} N_{k}(Y) h_{j}(Y) d \sigma(Y)$.

Clearly, $\tilde{\pi}=\pi+c$ since $\nabla \tilde{\pi}=\nabla \pi$. Note that $w_{k}$ is harmonic in $\Omega$ and $\left(w_{k}\right)^{*} \in L^{2}(\partial \Omega)$. By the area integral estimates for the harmonic function [D],

$$
\begin{aligned}
\int_{\Omega}|\tilde{\pi}|^{2} \delta(X) d X & \leq \sum_{k=1}^{n} \int_{\Omega}\left|\nabla w_{k}\right|^{2} \delta(X) d X \leq C \sum_{k=1}^{n} \int_{\partial \Omega}\left|\left(w_{k}\right)^{*}\right|^{2} d \sigma \\
& \leq C \int_{\partial \Omega}|h|^{2} d \sigma \leq C \int_{\partial \Omega}|u|^{2} d \sigma
\end{aligned}
$$

Also, since $\Delta \tilde{\pi}=0$ in $\Omega$, by interior estimates,

$$
\int_{\Omega}|\nabla \tilde{\pi}(X)|^{2} \delta^{3}(X) d X \leq C \int_{\Omega}|\tilde{\pi}(X)|^{2} \delta(X) d X \leq C \int_{\partial \Omega}|u|^{2} d \sigma .
$$

This completes the proof.

We are now in a position to give the:

Proof of Theorem A.1. Fix $P \in \partial \Omega$ and $r>0$ small. Using linear transformations both in the variable $X$ and functions $u, \pi$ (see [S1, p. 347]), we may assume that

$$
\Omega \cap B(P, 3 r)=\left\{(x, y) \in \mathbb{R}^{n}: y>\psi(x)\right\} \cap B(P, 3 r) .
$$

Let $\eta \in C_{0}^{\infty}(B(P, 2 r))$ such that $\eta \equiv 1$ on $B(P, r)$. Since $\Delta u=\nabla \pi$, $\operatorname{div} \pi=0$ in $\Omega$, we have

$$
\begin{aligned}
\Delta(u \eta) & =(\Delta u) \eta+2 \nabla u \cdot \nabla \eta+u \cdot \Delta \eta \\
& =(\nabla \pi) \eta+2 \nabla u \cdot \nabla \eta+u \cdot \Delta \eta \\
& =\nabla(\pi \eta)-\pi \nabla \eta+2 \nabla u \cdot \nabla \eta+u \cdot \Delta \eta \\
& =\nabla(\pi \eta)-f
\end{aligned}
$$

where $f=\pi \nabla \eta-2 \nabla u \cdot \nabla \eta-u \cdot \Delta \eta$.

Note that $|f| \leq C\{|\pi|+|\nabla u|+|u|\}$. Also, $\operatorname{div}(u \eta)=u \cdot \nabla \eta \equiv g$ and $|g| \leq$ $C|u|$. Moreover, it is not hard to see that

$$
|\Delta g+\operatorname{div} f| \leq C\{|\nabla u|+|\nabla \pi|+|\pi|+|u|\}
$$


We now apply Lemma A.3 to the equations

$$
-\Delta(u \eta)+\nabla(\pi \eta)=f, \quad \operatorname{div}(u \eta)=g
$$

in $D=\left\{(x, y) \in \mathbb{R}^{n}: y>\psi(x)\right\}$. Let $D_{r}=\Omega \cap B(P, r)$. We obtain

$$
\begin{aligned}
& \int_{D_{r}}|\nabla u|^{2} \delta(X) d X \leq C \int_{\partial \Omega}|u|^{2} d \sigma+C \int_{\Omega}|\pi|^{2} \delta(X) d X+C \int_{\Omega}|\nabla \pi|^{2} \delta^{3}(X) d X \\
&+C \int_{\Omega}|\nabla u||u| \delta^{2}(X) d X+C \int_{\Omega}|u|^{2} d X \\
& \leq C \int_{\partial \Omega}|u|^{2} d \sigma,
\end{aligned}
$$

where we have used Lemma A.9 in the second inequality. It follows that

$$
\int_{\Omega}|\nabla u|^{2} \delta(X) d X \leq C \int_{\partial \Omega}|u|^{2} d \sigma .
$$

To finish the proof, note that Lemma A.3 also gives

$$
\begin{aligned}
\int_{\partial \Omega \cap B(P, r)}|u|^{2} d \sigma \leq \varepsilon \int_{\partial \Omega}\left|(u)^{*}\right|^{2} d \sigma+C_{\varepsilon} & \left\{\int_{\Omega}|\nabla u|^{2} \delta(X) d X+\int_{\Omega}|u|^{2} d X\right. \\
& \left.\int_{\Omega}|\pi|^{2} \delta(X) d X+\int_{\Omega}|\nabla \pi|^{2} \delta^{3}(X) d X\right\} .
\end{aligned}
$$

It then follows from the $L^{2}$-estimates $[\mathrm{FKV}]$ that

$$
\int_{\partial \Omega}|u|^{2} d \sigma \leq C\left\{\int_{\Omega}|\nabla u|^{2} \delta(X) d X+\int_{\Omega}|\pi|^{2} \delta(X) d X+\int_{\Omega}|u|^{2} d X\right\} .
$$

This estimate holds for any pressure term $\pi$. In particular, we may choose $\pi$ such that $\pi\left(X_{0}\right)=0$ for some $X_{0} \in \Omega$. Then, by the Hardy inequality [St, p. $272]$,

$$
\begin{aligned}
\int_{\Omega}|\pi|^{2} \delta(X) d X & \leq C \int_{\Omega}|\nabla \pi|^{2} \delta^{3}(X) d X \leq C \int_{\Omega}\left|\nabla^{2} u\right|^{2} \delta^{3}(X) d X \\
& \leq C \int_{\Omega}|\nabla u|^{2} \delta(X) d X,
\end{aligned}
$$

where the last inequality follows from the interior estimates.

Thus,

$$
\int_{\partial \Omega}|u|^{2} d \sigma \leq C\left\{\int_{\Omega}|\nabla u|^{2} \delta(X) d X+\int_{\Omega}|u|^{2} d X\right\} .
$$

The proof is complete. 


\section{REFERENCES}

[BL] J. Bergh \& J. LÖFström, Interpolation Spaces, An Introduction, SpringerVerlag, Berlin, 1976.

[CF] P. Constantin \& C. Foias, Navier-Stokes Equations, Univ. of Chicago Press, 1988.

[D] B. DAHLBERG, Weighted norm inequalities for the Lusin area integral and the nontangential maximal functions for harmonic functions in a Lipschitz domain, Studia Math. 67 (1980), 297-314.

[DKPV] - C. Kenig, J. Pipher \& G. VerchotA, Area integral estimates and maximum principles for higher order elliptic equations and systems on Lipschitz domains, (Preprint).

[DW] P. Deuring $8 \mathrm{~W}$. VON WAHL, Strong solutions of the Navier-Stokes system on Lipschitz bounded domains, Math. Nachr. 171 (1995), 111-148.

[F] E. FABES, Layer potential methods for boundary value problems on Lipschitz domains, Lecture Notes in Math. 1344 (1988), 55-80.

[FKV] , C. Kenig \& G. Verchota, The Dirichlet problem for the Stokes system on Lipschitz domains, Duke Math. J. 57 (1988), 769-793.

[FK] H. Fujita $\&$ T. KATO, On the Navier-Stokes initial value problem, Arch. Rat. Mech. Anal. 16 (1964), 269-315.

[Gi] M. Giaquinta, Multiple Integrals in the Calculus of Variation and Nonlinear Elliptic Systems, Princeton Univ. Press, 1983.

[GT] D. Gilbarg 8 N. S. Trudinger, Elliptic Partial Differential Equations of Second Order, 2nd Edition, Springer-Verlag, 1983.

[Gr] P. Grisvard, Elliptic Problems in Nonsmooth Domains, Pitman, 1985.

[H] J. G. HEYWOOD, The Navier-Stokes equations: on the existence, regularity and decay of solutions, Indiana Univ. Math. J. 29 (1980), 639-681.

[JK] D. JERISON 8 C. KEnIG, The inhomogeneous Dirichlet problem in Lipschitz domains, J. Functional Analysis 130 (1995), 161-219.

[S1] Z. SHEN, Boundary value problems for parabolic Lamé systems and a nonstationary linearized system of Navier-Stokes equations in Lipschitz cylinders, Amer. J. Math. 113 (1991), 293-373.

[S2] - A note on the Dirichlet problem for the Stokes system in Lipschitz domains, Proc. Amer. Math. Soc. 123(3) (1995), 801-811.

[St] E. SteIn, Singular integrals and differentiability properties of functions, Princeton Univ. Press, 1971.

The authors were supported in part by the NSF. The first named author also received support from the Commonwealth of Kentucky through the NSF-EPSCoR program.

Department of Mathematics

University of Kentucky

Lexington, KY 40506

Received: July 3rd, 1995; revised: December 7th, 1995. 